\title{
On the electrodynamics of partial discharges in voids in solid dielectrics
}

\author{
Pedersen, Aage
}

Published in:

Proceedings of the 3rd International Conference on Conduction and Breakdown in Solid Dielectrics

Link to article, DOI:

10.1109/ICSD.1989.69171

Publication date:

1989

Document Version

Publisher's PDF, also known as Version of record

Link back to DTU Orbit

Citation (APA):

Pedersen, A. (1989). On the electrodynamics of partial discharges in voids in solid dielectrics. In Proceedings of the 3rd International Conference on Conduction and Breakdown in Solid Dielectrics (pp. 107-116). IEEE. https://doi.org/10.1109/ICSD.1989.69171

\section{General rights}

Copyright and moral rights for the publications made accessible in the public portal are retained by the authors and/or other copyright owners and it is a condition of accessing publications that users recognise and abide by the legal requirements associated with these rights.

- Users may download and print one copy of any publication from the public portal for the purpose of private study or research.

- You may not further distribute the material or use it for any profit-making activity or commercial gain

- You may freely distribute the URL identifying the publication in the public portal 
ON THE ELECTRODYNAMICS OF PARTIAL DISCHARGES IN VOIDS IN SOLID DIELECTRICS

A. Pedersen

\section{INTRODUCTION}

Partial discharges in voids in a solid dielectric are often discussed in terms of a simple equivalent capacitive circuit known as the abc-model. The vold is represented by a capacitance and discharges are simulated as discharges of this capacitance, see Whitehead [1]. An equivalent circuit is a system which can generate as faithfully as possible the signals which are manifest at the terminals of the actual system, and in this respect the abc-model has been quite successful. It is, however, important to realize that the processes within an equivalent circuit need not in any way be identical to the processes which are involved in the real system. This is evident for the abc-model since this model describes a phenomenon, which is inherently a field problem, in terms of lumped circuit parameters.

To envisage a vold as a capacitor requires a very loose interpretation of the concept of capacitance. This concept is intimately related to conducting electrodes between which a space-charge free electrostatic field can be established. This means that the field must be Laplacian. Strict proportionality will consequently exist between the charge $Q$ on the electrode and the applied voltage $U$, i.e.

$$
Q=C U,
$$

where $C$ is the capacitance. A meaningful application of equation (1) to the field within a void is hardly possible since the field is a Poisson-field when space-charges are present, and this implies that $Q$ will not be proportional to $U$.

The transients which are manifest at the electrodes of a system during partialdischarge activity are related to the charges which, in view of Faraday's icepail experiment, are induced on the electrodes. The primary sources for these induced-charges are the charges which, as a result of the partial-discharge activity, are distributed within the volds.

The induced-charge can be expressed as the difference between the charge on the electrode when discharges have occurred and the charge which would have been on the electrode had the system remained discharge free, Repp et al. [2]. The direct implementation of this approach can be rather cumbersome as it requires the solution of Poisson's equation. A more stralghtforward approach is possible through an application of the principle of super-position. This can be done in two different ways depending on whether the analysis is based on the D-field, Pedersen [3], Crichton et al. [4], or on the P-field, Pedersen [5]. The former, the Maxwellian description, is more convenient for practical applications, whereas the latter, the quasi- molecular description, is suitable for fundamental studies of the physics of the phenomenon.

A. Pedersen is with Physics Laboratory II, The Technical University of Denmark, Building 309B, DK-2800 Lyngby, Denmark. 
THE CONCEPT OF INDUCED CHARGES

Maxwellian Description

The charge $Q_{1}$ on the $1^{\prime}$ th electrode in a space-charge free system consisting of $\mathrm{N}$ electrodes is given by

$$
Q_{i}=\sum_{j=1}^{N} c_{i j}\left(U_{i}-U_{j}\right)
$$

with

$$
\sum_{i=1}^{N} Q_{1}=0 .
$$

$U_{i}$ and $U_{j}$ are the potentials of the $i^{\prime}$ th and the $j^{\prime}$ th electrodes, and $C_{i j}$ is the partial capacitance between these electrodes. If space-charges are present in the space between the electrodes an additional charge $q_{i}$, the induced charge, will appear on the $i$ 'th electrode, i.e.

$$
Q_{i}=q_{i}+\sum_{j=1}^{N} c_{1 j}\left(U_{1}-U_{j}\right) .
$$

The induced-charge depends in a unique way on the locations and magnitudes of the space-charges. They are independent of the electrode potentials if the permittivities do not depend on the electric field. An infinitesimal charge dQ located somewhere between the electrodes will induce a charge $d q_{i}$ on the $i$ 'th electrode and $\mathrm{d} q_{i}$ will, in view of the principle of superposition, be proportional to dQ, i.e.

$$
\mathrm{d} q_{i}=-\lambda_{i} \mathrm{~d} Q
$$

The parameter $\lambda_{i}$ is a dimensionless positive scalar function which depends on the location of $d Q$ only. The entire induced-charge on the $i$ th electrode from a distribution of space charges can thus be expressed in the form

$$
q_{1}=-\iiint \lambda_{i} \rho \mathrm{d} \Omega-\iint \lambda_{1} \sigma \mathrm{d} S
$$

in which $\rho$ is the volume-charge density in the volume element $\mathrm{d} \Omega$ and $\sigma$ is the surface-charge density on the surface element d $S$ of an interface between two dielectrics. The volume integral is extended over all space and the surface integral over all dielectric interfaces. In a study of partial discharges in voids in solid dielectrics the space-charges will be located within the voids and on the walls of these voids.

The response function $\lambda_{i}$ can, as shown by Maxwell [6], be found by applying Green's reciprocal theorem to the system in the following way. The total charge on the $i$ 'th electrode will be $q_{i}$ if all electrodes are at zero potential, see equation (4), and space-charges are deposited in the space between the electrodes. We compare this with the situation when $\rho=0$ and $\sigma=0$ everywhere, the potential of the $i$ 'th electrode is $U_{i}$ and all other electrodes are at zero potential. Applying Green's reciprocal theorem, clemmow [7], to these two situations yields 


$$
\begin{aligned}
& U_{i} q_{1}+\iiint v_{i} \rho \mathrm{d} \Omega+\iint v_{i} \sigma \mathrm{d} S=0 . \\
& q_{i}=-\iiint\left(v_{i} / U_{1}\right) \rho \mathrm{d} \Omega-\iint\left(v_{i} / U_{i}\right) \sigma \mathrm{d} S .
\end{aligned}
$$

$V_{1}$ denotes the scalar potentials at $\mathrm{d} \Omega$ and $\mathrm{d} S$ when the $i$ th electrode is at the potentis $U_{1}$, all other electrodes are at zero potential and the system is space-chare free. Comparing (6) and (8) shows that

$$
\lambda_{1}=v_{1} / U_{1} \text {. }
$$

Since $V_{1}$ is the solution to Laplace's equation

$\operatorname{div}\left(\epsilon \operatorname{grad} V_{i}\right)=0$

$\lambda_{i}$ can be determined from

$$
\operatorname{div}\left(\epsilon \operatorname{grad} \lambda_{i}\right)=0
$$

in which $\epsilon$ denotes the permittivity. The boundary conditions are $\lambda_{1}=1$ at the surface of the $i$ 'th electrode and $\lambda_{1}=0$ at the surfaces of all the other electrodes. In addition, the following condition must be fulfilled at all dielectric interfaces such as the walls of the voids, viz.

$$
\epsilon_{+}\left(\partial \lambda_{i} / \partial n\right)_{+}=\epsilon_{-}\left(\partial \lambda_{i} / \partial n\right)_{-}
$$

where $\lambda_{i}$ is differentiated in the direction normal to the interface and the signs $t$ and - refer to the two sides of the interface. Since equation (11) is Laplace's equation any standard method for the calculation of space- charge free electrostatic fields can be used to evaluate $\lambda_{i}$.

\section{Quasi-Molecular Description}

The polarization $P$ in the dielectrics is a significant property when discussing the induced charge in a system which contains polarizable materials. The importance of the polarization is, however, not evident from the analysis given above since the effect of the polarization is automatically included in the $\lambda$ function. This is an advantage when the analysis is applied to practical systems whereas it may be less suitable in studies of the physics of the phenomenon.

The effect of the polarization $P$ can be taken into account by adopting a quasimolecular description [5]. This means that the entire space between the electrodes is viewed as vacuum in which the dielectric is represented by distribution of dipoles with a dipole moment density equal to $P$. The inducedcharge on an electrode is then considered to consist of two parts. One which is linked with the space-charge distribution created by partial discharge activities, and another part which is related to the dipoles, that is to the polarization $P$.

The induced charge related to a dipole can be found by visualizing a dipole as two charges $Q$ and $-Q$ separated by an infinitesimal distance $\mathrm{d} \vec{r}$. The dipole moment thus becomes

$$
d \vec{\mu}=Q d \vec{r} .
$$


Let $\varphi$ denote the $\lambda$-function for vacuum, then

$$
\varphi_{i}(\vec{r}+\mathrm{d} \vec{r})=\varphi(\vec{r})+\mathrm{d} \vec{r} \cdot \operatorname{grad} \varphi_{i},
$$

where $\vec{r}$ is the radius-vector indicating the position of the dipole. The inducedcharge on the $i^{\text {th }}$ electrode thus becomes

$$
\mathrm{d} q_{i}=-\mathrm{d} \vec{\mu} \cdot \operatorname{grad} \varphi_{i} \text {. }
$$

The response function for vacuum $\varphi_{1}$ is given by Laplace's equation for vacuum, i.e.

$$
\operatorname{div} \operatorname{grad} \varphi_{1}=0
$$

or

$$
\nabla^{2} \varphi_{1}=0
$$

The boundary conditions are $\varphi_{i}=1$ at the surface of the $i$ 'th electrode and $\varphi_{i}$ - $O$ at all other electrodes. No condition is imposed on $\varphi_{1}$ at the dielectric interfaces. Any svailable method of electrostatic field calculation in vacuum can thus be applied to obtain $\varphi_{i}$ from the equations

$$
\nabla^{2} V_{v i}=0
$$

and

$$
\varphi_{1}=v_{v i} / U_{1} .
$$

$V_{v 1}$ is the scalar potential in a point of the space-charge free electrostatic field in vacuum where the potential of the $i$ 'th electrode is $U_{1}$ and all other electrodes are at zero potential.

The polarization $P$ in a point of the solld dielectric will depend on the applied voltages and on the space-cherges formed by partial discharges, $1 . e$.

$$
\vec{P}=\vec{P}_{a}+\vec{P}_{\mathrm{g}} \text {, }
$$

where $P_{\mathrm{a}}$ is linked with the applied voltages and $P_{\mathrm{B}}$ is related solely to the inter-electrode space-charges. The resulting induced-charge on the $i$ 'th electrode as a result of partial discharge activitles will thus be given by

$$
q_{1}=-\iiint\left[\varphi_{1} \rho+\vec{P}_{8} \cdot \operatorname{grad} \varphi_{1}\right] \mathrm{d} \Omega-\iint \varphi_{1} \sigma \mathrm{d} S .
$$

The volume integral is extended over the entire space between the electrodes and the surface integral over all dielectric interfaces, that is the walls of the volds.

CURRENT PULSES IN THE LEADS

During the periods of time in which partial discharges are developing transient currents can flow in the leads to the electrodes and voltage transients can be observed. In addition a transfer of charge may occur from an electrode to the space between the electrodes. This is likely to appear if a vold is located at an interface between an electrode and the solid dielectric. 
Let $I_{i}$ denote the total current flowing in the lead towards the $i$ 'th electrode and let $I_{t i}$ be the current which represents the transfer of charge from the 1 'th electrode towards the inter-electrode space. These currents are, in view of the principle of the conservation of charges, related to the total net charge $Q_{i}$ on the $i$ 'th electrode in the following way

$$
I_{i}=I_{\mathrm{ti}}+\mathrm{d} Q_{\mathrm{i}} / \mathrm{dt}
$$

or

$$
I_{i}=I_{t i}+\frac{\mathrm{d} q_{1}}{\mathrm{~d} t}+\sum_{j=1}^{\mathrm{N}} c_{1 j}\left[\frac{\mathrm{d} U_{1}}{\mathrm{~d} t}-\frac{\mathrm{d} U_{1}}{\mathrm{~d} t}\right] .
$$

Differentiating equation (6) with respect to the time $t$ yields

$$
\frac{\mathrm{d} q_{1}}{\mathrm{~d} t}=-\iiint \lambda_{i} \frac{\partial \rho}{\partial t} \mathrm{~d} \Omega-\iint \lambda_{i} \frac{\partial \sigma}{\partial t} \mathrm{~d} S
$$

which by means of the continuity equations

$$
\operatorname{div} \vec{J}+\frac{\partial \rho}{\partial t}=0 \text { and Div } \vec{J}+\frac{\partial \sigma}{\partial t}=0
$$

can be written in the form

$$
\frac{\mathrm{d} q_{1}}{\mathrm{~d} t}-\iiint \lambda_{i} \mathrm{div} \vec{J} \mathrm{~d} \Omega+\iint \lambda_{1} D i \mathrm{v} \vec{J} \mathrm{~d} S .
$$

$\vec{J} 18$ the current density in a point within a void during discharge activities. Div $\vec{J}$ is the interface divergence defined in the following way, Fischer [8].

$$
\text { Div } \vec{J}=\vec{n} \cdot\left(\vec{J}_{+}-\vec{J}_{-}\right)
$$

where the signs + and - refer to the two sides of the interface, and $\vec{n}$ is a unit vector normal to the interface and directed away from the positive side. It 18 in this analysis assumed that the effect of the surface conductivity of the interfaces, 1.e. the walls of volds, can be neglected. Introducing the identities

$$
\operatorname{div}\left(\lambda_{1} \vec{J}\right)=\lambda_{1} \operatorname{div} \vec{J}+\vec{J} \cdot \operatorname{grad} \lambda_{1}
$$

and

$$
\lambda_{1} \operatorname{Div} \vec{J}-\operatorname{Div}\left(\lambda_{1} \vec{J}\right)
$$

then leads to

$$
\frac{d q_{1}}{d t}=\iiint \operatorname{div}\left(\lambda_{1} \vec{J}\right) d \Omega+\iint \operatorname{Div}\left(\lambda_{1} \vec{J}\right) d s-\iiint \vec{J} \cdot \operatorname{grad} \lambda_{1} d \Omega .
$$

Applying the extended divergence theorem of Gauss, Joos [9], to the vector field $\lambda_{1} J$ reveals that

$$
I_{\mathrm{t} 1}+\iiint \operatorname{div}\left(\lambda_{1} \vec{J}\right) \mathrm{d} \Omega+\iint \operatorname{Div}\left(\lambda_{1} \vec{J}\right) \mathrm{d} s=0 .
$$


This means that

$$
\frac{\mathrm{d} q_{1}}{\mathrm{~d} t}=-I_{\mathrm{ti}}-\iiint \vec{J} \cdot \operatorname{grad} \lambda_{1} \mathrm{~d} \Omega .
$$

Inserting this in equation (23) shows that the total current flowing in the lead towards $1^{\prime}$ th electrode is given by

$$
I_{i}=-\iiint \vec{J} \cdot \operatorname{grad} \lambda_{i} \mathrm{~d} \Omega+\sum_{j=1}^{N} c_{i j}\left[\frac{\mathrm{d} U_{i}}{\mathrm{~d} t}-\frac{\mathrm{d} U_{\mathrm{j}}}{\mathrm{d} t}\right] \text {. }
$$

Since the $\lambda_{i}$-function is determined by Laplace's equation, see equation (11), the gradient of $\lambda_{i}$ can be found from

$$
\operatorname{grad} \lambda_{i}=-\vec{E}_{i} / U_{i},
$$

in which $\vec{E}_{j}$ is the field strength in the space-charge free electrostatic field between the electrodes of the system if the potential of the $i$ 'th electrode is $U_{i}$ and all other electrodes are at zero potential. It should be emphasized that $U_{i}$ and $E_{1}$ are entirely fictitious quantities since $U_{i}$ can be given any arbitrarily chosen value in the computational procedure, 1.e. $U_{1}$ is not synonymous with the potential of the $i$ th electrode during discharge activities. For this reason $E_{i}$ and $U_{i}$ should not be inserted in the expression for the current.

Should it be desirable to include the polarization in the analysis a similar procedure can be applied to equation (21). This leads to the following expression for the total current in the lead to the $i$ 'th electrode.

$$
I_{\mathbf{i}}=-\iiint\left\{\left[\vec{J}+\frac{\partial \vec{P}_{\mathrm{s}}}{\partial t}\right] \cdot \operatorname{grad} \varphi_{i}\right\} \mathrm{d} \Omega+\sum_{j=1}^{N} c_{i j}\left[\frac{\mathrm{d} U_{i}}{\mathrm{~d} t}-\frac{\mathrm{d} U_{\mathrm{j}}}{\mathrm{d} t}\right] .
$$

The gradient of $\varphi_{i}$ can be calculated from

$$
\operatorname{grad} \varphi_{1}=-\vec{E}_{\mathrm{vi}} / U_{i} .
$$

where $E_{\mathrm{v} i}$ is the fleld strength in the electrostatic field associated with $U_{j}$ when the entire space between the electrodes is vacuum and all other electrodes are at zero potential.

The current density $J$ is related to the motion of electrons and ions within the voids during discharge activities, viz.

$$
\vec{J}=\sum_{k=0}^{\mathrm{m}} \rho_{\mathrm{k}} \vec{u}_{\mathrm{k}}
$$

where $\rho$ is the charge density and $\vec{u}$ the drift velocity, $k=0$ refers to electrons and $m$ is the number of possible species of positive and negative ions which are participating in the discharge activity.

A direct application of equations (21) and (35) to practical systems would be rather complicated because of the explicit occurrence of the polarization in these equations. A closer analysis, see Pedersen [5], shows that the polarization does play an important role, and that this effect becomes more dominant the more oblate the vold is with respect to the direction of the 
applied electric fleld.

Formulae similar to those derived above, but for systems in which polarizable materials are absent, have been given by many authors, see for example von Engel and Steenbeck [10], Ramo [11], Shockley [12]. These formulae are sometimes referred to as the Ramo-Shockley theorem. It should be remembered, however, that a quantitative treatment based on Faraday's concept of induced charges dates back at least to Maxwell [6].

Although the analysis given above is discussed with special reference to partial discharges in voids, the sets of formulae are of general validity for similar systems in which currents in the external leads depend on charges in motion in the space between the electrodes.

\section{APPLICATION TO PARTIAL DISCHARGES}

A discharge in a void results in a deployment of charges on the surface $s$ of the vold. The surface-charge density $\sigma$ will attain such values that the electric field within the vold will reduce untll the discharge is quenched. Since the detection of partial discharges often refers to signals which are manifest at the terminal electrode we can leave out the subscripts in the following discussion. The resulting induced-charge $q$ on this terminal electrode 15 , in view of equation ( 6 ), given by

$$
q=-\iint_{S} \lambda \sigma \mathrm{d} S
$$

The dimensions of the void are normally small relative to the distance to the electrode, and the nett charge contained within the vold will remain zero. This means that the charges which are deposited on the wall $S$ of the void can be considered, to a first approximation, as an electric dipole configuration with the dipole moment

$$
\vec{\mu}=\iint_{S} \vec{r} \sigma \mathrm{d} S
$$

where $\vec{r}$ is a radius vector which locates the position of the surface element dS. The induced-charge which arises from this dipole is given by, see equation (15).

$$
q=-\vec{\mu} \cdot \operatorname{grad} \lambda \text {. }
$$

In general the gradient of $\lambda$ can be determined only in such cases where the location and the geometrical form of the void are known. This difficulty can to some extent be circumvented by replacing $\lambda$ with the value which the $\lambda$-function would attain if we assume that the entire insulating system is completely free from any voids. Let $\lambda_{0}$ denote this idealized function. The gradients of $\lambda$ and $\lambda_{0}$ are connected by

$$
\operatorname{grad} \lambda=h \operatorname{grad} \lambda_{0} .
$$

If the application is restricted to simple geometries, such as spheroids, we can consider the parameter $h$ to be a scalar. Based on the mathematical analogies between the $\lambda$-function and the electrostatic field it is easily seen that 


$$
1<h<\epsilon_{\mathrm{r}}
$$

where $\epsilon r$ is the relative permittivity of the amblent dielectric. The lower limit applies to voids which are very prolate with respect to the direction of the applied electric field. The upper limit is approached for a very oblate void. Introducing $\lambda_{0}$ leads to the following expression for the induced charge

$$
q=-h \vec{\mu} \cdot \operatorname{grad} \lambda_{0} \text {. }
$$

An assessment of the dipole moment requires a knowledge of the shape and location of the void. The nature of the gas within the void must also be known. A quantitative analysis related to ellipsoidal voids has been given by Crichton et al. [4].

All the required data will not be available in connection with partial discharge testing of commercial high voltage equipment. The dipole moment and the parameter $h$ will, however, remain constant if we consider discharges in a number of voids of fixed volume and form containing the same gas, but placed at different locations within the insulating system. The induced- charge will in such cases vary with the location of the void in the same way as the gradient of $\lambda_{0}$ or, in view of the analogy with the electrostatic field, as indicated by the variation of the electric field strength for the idealized void-free system.

\section{TRANSIENTS RELATED TO INDUCED-CHARGES}

Although the observable transients are inherently related to the inducedcharges, the properties which primarily are measured are transients in the applied-voltage and current-pulses in the lead to the terminal electrode. The relationship between these properties and the induced-charge can be found in the following manner. Just prlor to the first discharge the potential of the electrode is $U$ and the associated charge on the electrode is $Q$. We compare this with the situation immediately after the discharge is quenched. The potential has now dropped to $U-\Delta U$ and the charge on the electrode has become $Q+\Delta Q$, where $\Delta Q$ is the charge transferred to the electrode from the external source. Green's reciprocal theorem then yields

$$
(U-\Delta U) Q=U(Q+\Delta Q)+\iint \operatorname{Vod} S \text {, }
$$

where $V$ is the scalar potential at the surface element ds for the dischargefree situation. Since $V=\lambda U$ in consequence of equations (11) and (12), and $Q=$ $C U$, where $C$ is the capacitance of the system, we obtain

$$
-\iint_{S} \lambda \sigma d s=C \Delta U+\Delta Q
$$

$$
q=C \Delta U+\Delta Q
$$

If the impedance of the circuit is large for the current which is associated with the discharge, then $C \Delta U$ can be much larger then $\Delta Q$. The induced-charge is then given by approximately

$$
q=c \Delta U
$$

It should be emphasized that the capacitance of the system is not affected by 
DISCHARGE PHENOMENA IN VOIDS FILLED WITH FIBROUS MATERIALS

P.H.F. Morshuis, F.H. Kreuger

ABSTRACT

Untill now paper 3-phase belt type cables were succesfully used for voltages upto $20 \mathrm{kV}$ in great parts of the world. Replacement of paper by extruded insulation has been unsuccesfull and service voltages no higher than $5 \mathrm{kV}$ can be applied because of the large discharges and uncontrolled field in the interstices at the core and the belt. Belt-type cables remained paper-insulated for that reason.

A breakthrough has been obtained when a non-woven fibrous tape was applied to the cores to improve the longitudinal water tightness. As these tapes are very poor dielectrics, with low breakdown stress, it was expected that the breakdown tests would be impaired. At the contrary, the life expectancy was raised from less than 10 years to more than 30 years at $10 \mathrm{kV}$. Further study revealed that this dielectric improvement was caused by a considerable reduction of the discharges in the interstices of the cable; reductions of several hundred times were measured.

\section{INTRODUCTION}

To obtain a waterstop in the longitudinal direction of a 3-phase belted cable, a thin non-woven tape was applied to the core insulation and to the interstices between the belt insulation and the core insulation (Figure 1), Kreuger, Morshuis et al. [1]. The discharge diagram of this cable and a non-taped cable are shown in Figure 2. The research on the discharge mechanism of voids filled with a nonwoven, fibrous tape was initiated by the discharge limiting effect of the tape in the cable and by the fact that the cable life was significantly increased.

To study the discharge mechanism of these taped cables a model was built that represented the interstices in the cable.

EXPERIMENTAL METHOD

The testcircuit for measuring partial discharges consists of a 50 Hz high voltage source that can deliver a programmable number of half or whole $50 \mathrm{~Hz}$ periods. This set-up makes it possible to study subsequent discharges or Lichtenberg figures during specific time intervals. A balanced discharge detection system is used (Figure 3 ) combined with a storage facility. Lichtenberg figures can be

P.H.F. Morshuis is with the High Voltage Laboratory of the Technical University of Delft, The Netherlands.

F.H. Kreuger is professor of High Voltage Technology at the Technical University of Delft, The Netherlands. 
not prohibit a sound qualitative assessment of the insulation to be made on the basis of such measurements.

CONCLUSION

A correct interpretation of partial-discharge transients can be attained only through the concept of induced-charge. The application of this concept has enabled a partial-discharge theory to be developed through which the influence of relevant void parameters can be assessed in a correct way. In contrast, the widely adopted abc-capacitance model does not allow this insight to be achieved. In addition, the $a b c$-model is based on an erroneous application of the concept of capacitance, and, although this simple approach can be a useful tool when discussing measuring techniques, it may lead to quite incorrect conclusions.

\section{ACKNOWLEDGMENT}

The author wishes to thank the Electric Power Research Institute for supporting this work through project RP2669-1.

\section{REFERENCES}

1. Whitehead, S., 1951, "Dielectric Breakdown of Solids", Clarendon Press, Oxford, England.

2. Repp, H., Nissen, K.W., and Röhl, P., 1983, Siemens Forsch.- u.Entwickl. -Ber., 12, 101-106.

3. Pedersen, A., 1987, 1987 Annual Report - Conference on Electrical Insulation and Dielectric Phenomena, IEEE Publication 87CH2462-0, 58-64.

4. Crichton, G.C., Karlsson, P.W., and Pedersen, A., 1989, IEEE Transaction on Electrical Insulation, 24, April Is sue.

5. Pedersen, A., 1986, IEEE International symposium on Electrical Insulation, IEEE Publication 86CH2196-4-DEI, 112-114.

6. Maxwell, J.C., 1873, "A Treatise on Electricity and Magnetism", 1 , Clarendon Press, Oxford, England.

7. Clemmow, P.C., 1973, "An Introduction to Electromagnetic Theory", Cambridge University Press, Cambridge, England.

8. Fischer, J., 1976, "Elektrodynamik", Springer-Verlag, Berlin, Federal Republic of Germany.

9. Joos, G., 1934, "Theoretical Physics", Blackie and Son, Glasgow, Scotland. Also available as a Dover Publication.

10. Engel, A. v., and Steenbeck, M., 1932, "Elektrische Gasentladungen", 1 , Springer-Verlag, Berlin, Germany.

11. Ramo, S., 1939, Proc. I.R.E., 27, 584-585.

12. Shockley, W., 1938, J. App1. Phys., 9, 635-636. 